\title{
Online Video Editing and Production Skills*
}

\author{
Xiaoyan Lu \\ College of Media and International Culture, Zhejiang University, Hangzhou, China \\ Email: xinwen1@zju.edu.cn
}

Received April 12, 2012; revised May 13, 2012; accepted May 22, 2012

\begin{abstract}
Internet video is a video service that can be uploaded on the Internet and played online. As online video post-production of the key aspects related to the editing work is often the final stage of artistic effects, the acquisition of a certain amount of online video editing skills is important. It will make people relatively independent of the picture and sound that mixed together organically, and be more conducive to the recognition of formation system, the color of the web video and audio clips.
\end{abstract}

Keywords: Online Video; Internet; Editing Skills

\section{Introduction}

Internet video is a video service that can be uploaded on the Internet and played online. Online video post production of the key aspects related to the editing work is often the final stage of the artistic effects. Nowadays, the nonlinear editing system is more commonly used on network video material and is combined with 3DSMAX, Aftereffect, Photoshop, Flash, Authorware and other software synthesis technology to highlight the power of Internet video’s performance and artistic charm.

\section{Montage Theory}

Montage video editing provides a theoretical basis for the network. A complete network video works by a number of plots is composed by paragraphs, and each paragraph of a plot consists of the montage of several paragraphs (or sentences montage). "Montage" is derived from the French, the original architectural term, meaning composition, assembly. Later become one of the main narrative and performance of its means on creating and editing video and audio synthesis. With the way of montage, video enjoys great freedom of space and time jumping. Montage editing relies on the intrinsic link to create the lens topic, which guides the audiences to interpret events and strengthens the events through the combination of image and sound. What's more, through the combination of ways, the director would be able to express his ideas.

\footnotetext{
*This research was sponsored by Key Research Base of Social Science: Zhejiang Social Sciences Research Center of Communication and Cultural Planning Issues, and also sponsored by Digital Future and Media Institute for Social Research of Zhejiang University. In this paper, results from Experimental teaching demonstration centers in ZhejiangMedia experiment teaching centre of Zhejiang University.
}

Internet video is a collection of the greatest features of voice, video, text and other elements of advantages, which can attract the audiences' attention within a short time. Therefore, it requires full utilization of this particular montage editing techniques, and left the audiences with space of imagination in the meantime.

It is difficult to have an exact accurate conclusion on the classification of montage. According to the processing view of means of montage, it can be divided into rhythmic montage, analytical montage, Lenovo montage, montage and other types of modeling; and according to the function, it can be divided into narrative montage, montage and rational expressive montage.

Rhythmic montage segment mainly for the screen of time, one is such a time slice, the other is the time frame or clip the situation gradually decreasing and increasing. Lenovo montage composes of different compared and conflict contents. Compared the images associated with one subject by two different groups received from the event or scene before and after montage, for the expression of two related feelings caused by a subject. The montage in the online video advertising is in the highest frequency of use, which makes up the main concept of the unity of the performance of concrete problems, reflecting the subtle handling of the ads enhanced the artistic expression of online video advertising.

Many lens in the online video advertising performance and the group are often different from the situation of a simple narrative, the performance of the content does not necessarily have strong logic, then the director or production division by modeling similar morphology, motion and psychological style trend similarity with the fast way to stack in series screen. We will shape this relationship due to the formation of art called montage style 
montage. Of course, this way of modeling montage production methods are generated from the perspective of the director which can be classified as associative montage, but feeling a bit far-fetched. It is always presented as performance-based shape modeling montage and structure similarity, such as curve similar to the direction, other art elements similar to the movement.

Montage is a unique way of thinking and artistic images, and the basic structure of the network video tools. It not only includes the lens, scenes, paragraphs, and all the arrangements with the combination of artistic skills, but also works in post-production and edition, and act as a guideline for certain internet staff work and audio material for the video.

\section{Online Video Editing}

In the non-linear editing work, the need for video performance, the action, mood, tempo editing point selection must follow the objective law, in line with the logical development of things, and meet people's thinking habits.

\subsection{Different Types of Online Video Editing}

A variety of show type video, most of the dance-based, and its clip point basis having their songs and music melody, rhythm, phrasing, music section to choose, and many strengths on the beat in the music switch lenses. TV type video, most of the development of the plot and characters depend on changes in emotional choose edit points. For network interview shows, usually those conversations and interviews with the atmosphere to switch lenses. Network feature documentaries and documentary video editing should strive to be authentic, especially the long shots, you should try to ensure the integrity of the lens to avoid the cut being too small or broken. Competitive sports video, with a strong dynamic, strong dynamic place should be selected as the switching point.

\subsection{Select the Length of the Video Camera}

In general, do not view the video camera, video screen and the number of information constitute the complexity of the screen will affect the choice of the length of the lens. On the Scene, the panoramic camera images to stay longer; the shorter number of close-range shot, close-up shorter; to screen information, the screen stays longer with amount of information, and shorter with less of information; to a complex picture extent, a complex picture stays longer, shorter and vice versa.

\subsection{Video Screen Editing Technique}

Errors in the lens during shooting can be compensated to some extent in post-production by using the sub-cut, cutout and fight a shot cut. Sub-cut is to cut a lens into two or more, cutout is a complete shot in the action to remove a part of the screen without generating a sense of jump. Fight a shot cut is repeated splicing, generally used in the lens case which is not long enough and can not Bupai (reshoot) before use. These editing techniques contribute to balance the video rhythm better, in which way the dramatic effect can be strengthened by the screen and fluency.

\section{Online Video Lens Group Received}

The network needed for exciting video montage down, the lens needs to be connected in coherence, for the audience to receive a better understanding of video content. The different lens combinations will lead to the transformation sequence of different artistic effects. In addition to footage of the group means of the optical principles, switching the camera directly through the convergence of law, the plot will be more natural and smooth.

\subsection{Video Camera Connected to a Logical Group}

Shot of the group is required to meet people's life experience and in line with the logic of thinking, which is the basis for the audience to read the video. Therefore, the lens group must consider the audiences' visual requirements, lead the audience in mental activity, we must also meet the visual enjoyment.

Video camera should follow the group and the law of "move then the dynamic and static access of static, dynamic and static phase to the transition". The action is consistent to achieve the purpose of smooth and simple transition; the previous screen done a full body movement then stops, connecting to a movement from rest to start the lens to achieve a "static access quiet".

\subsection{Shot Group then the Unity of Tone Color}

The tone color should maintain consistency, regardless of network video screen is black and white or color. If the connection between two lens groups is too brightness or with strong color contrast (except for special needs), people would feel awkward, stiff, non-coherent and without a clear expression of content.

\subsection{The Rhythm of the Video Camera Connected Transformation Group}

The subject matter, style, style and ambience, character emotions, plot progress and the rhythm of the lens group of network video receive a total basis. In addition to video rhythm performers, camera movement and transformation, the scene reflects the spatial and temporal changes with music and other factors outside the group need access to the use of means, which should strictly adhere to the size and number of the lens. Lens group 
should pay attention not on the seats or the King of mutual coordination and cooperation. If you encounter with the same flight, even with the Scene with the main screen, not directly connected group, this screen led screen similar lack of change in composition. But the picture changes a bit of a jump will make the audience an eye movement, destroying the continuity of images and coherence of thinking. Others such as the direction of the screen, the length of time controlled by camera, unity of color and tone, rhythm and harmony and other factors, and that video editing needs attention which strives to make editing a harmonious flow of network video screen. It is necessary to meet people's logical thinking and teaching law, which is also rich in artistic expression.

\section{Combination of Audio Editing and Sound and Picture}

Sound and picture are two important elements in network video, the two instruments working together allows online video to be viewed and listened. In network video, audio language with rich elements of performance of its forms and manifestations is able to add clear and accurate information to the visualized video. Therefore, the audiences can not only see and hear, but also feel. The term of Voices here refers to the concept which consists of three parts: language, music and sound. Language is communication and the primary means of transmitting information, logical, systematic and complete expression to people's thoughts and feelings, which includes dialogue, narration and monologue. Music, including instrumental and vocal music, often in online video in the form of voice-over, the atmosphere can help screen contrast, infection audience. Sound refers to all sound except human voice and music. It has a broad scope, including almost all kinds of sounds of the nature, such as wind, thunder, bark and so on. In network video, the audio mainly subordinates to the picture environment.

A video clip with non-linear editing is usually a combination of the two parts to be added to the time of selected material line, the audio files will automatically appear in the associated audio track to correspond with the associated video and audio. However, in network video editing process, you may only need a part of the video or audio, which requires cutting off the relationship between the two, also known as the melting operation. Through video and audio solutions chain operations, non-linear editing system can be decomposed into separate video and audio section, which can be moved and edited individually. Audio files and video files are handled in the same way, which is proceeded by relying on filters and switch techniques. If this software in Premiere, sound combinations are divided into three categories: sound comparison (meaning different sounds simultaneously, so that in contrast to the main sound produced in the contrasting roles), the sound of the coverage (in the same footage appear on a variety of similar sounds in parallel, there is a voice above the sound on the other, to draw attention to the attention of sound body), sudden turn of sound and silence (sound and silent alternating). Sound editing and processing include: play, clip, gain and gradual change, swing left and right channels and volume balance adjustment, and the usage of audio mixer (mixer).

Sound production and production are inextricably linked images, sounds act as bridge, so that the screen is connected smoothly. Rendering the sound is conducive to the psychological state of characterization and helps heighten atmosphere, so it still faces painting activities. Sound and images are together in a certain way, including the unity of sound and picture, sound and picture on the digital picture and sound separation of three types.

Combined with sounds, the correspondence created by sound and images and that between the pictures is in consistent with the audio content, which makes visual information and auditory information simultaneous, and meets audiences' needs and enhances picture realism in the meantime. Some networks, such as live, online news Tong Qisheng, etc., are generally sound and picture ecumenical relations. Sound and picture on the digital sound and pictures is to maintain the internal relations, but the sound and the picture is not one to one, they exists in their own separate basis. However, the organic combination of clips makes the meaning of sound in accordance with the contents of the screen, which can be interpreted from the overall performance of the video content. Science and education videos in the network, some description, analysis, explanatory language belongs to the relationship between sound and picture on the bit.

The immediately points of sound and picture are different, the relationship between the formation is of a completely opposite. The opposition and collision caused by sound and pictures would result in a strong artistic effect.

Sound and picture are complementary and mutually reinforcing, understanding and properly handling of relations between sound and picture can make sound and picture harmonious, and improve the performance of network video greatly.

\section{Online Video Editing Effects}

In the network often need to switch to video games, it allows the lens to achieve the natural switch to make the story more coherent and easy. The first is the transformation scene of the conversion between the lens which includs the montage and plot and the conversion between paragraphs transition between paragraphs. In order to make the network video content be more rational, level of development clearer, transition effect is in the transition between scenes in some way needed. 


\subsection{Fade in, Fade out}

Fade is a paragraph on the last shot until the black screen fading away, fading the next paragraph refers to the first screen shot gradually emerging from a black screen until the normal brightness, the entire conversion process was $\mathrm{V}$-changing visual stimuli. The length in the screen of fade out and is usually 2 seconds each, but the actual editing should be decided on the basis of TV series plot, mood, and rhythm. Light change is the most common performance of Internet video to achieve the conversion of natural passage with space and time interval techniques.

\subsection{Wipe}

Wipe, also known as programs, program changes, generally used for significant differences between the two large paragraphs of content conversion. Wipe can be divided and assigned to draw. The previous screen to exit from a screen called the draw direction, the direction of the next screen, enter the screen is called from a classified. Slip is the main performance characteristics, that is, a shot after the previous lens slowly across the screen. Planning approach, the direction of diversity, such as about planning, planning from top to bottom, draw polygon, prism program, such as spiral-shaped plan, according to the needs of plot development and the direction of motion picture use a certain form. Wipe the strengthening of visual effects, rendering emotions or reinforce the rhythm is very effective, but improper use of this technique is easy to produce stiff and artificial feeling.

\subsection{Dissolve Imaging}

Dissolve the video screen before and after a shot of a screen shot of the overlay of the picture before fading a shot until the disappearance of a shot after a clear picture of the gradual process until fully apparent. In the online video editing and production, the technology changes are mainly of the following functions: First, for the time of conversion, said the passage of time; the second is used for space conversion, said the space has changed; the third performance is the dream of change, imagination, memory and other plug Syria, back to Syria occasions; fourth, the performance of scene changing, dazzling, dizzying.

\subsection{Image Freeze}

Freezing the screen image in the last frame for some time gives rise to visual pause, emphasis and meaning with the visual impact of instant still screen. It contributes to highlight or render the scenes, image, demeanor, detail and so on.

\subsection{Keying Effects}

Keying effects through the control of electrical signal, pulling out part of the video image by filling with another image to produce a result of one screen embedded into another screen in a certain part. Images can be synthesized using keying effects, cannot be realized in daily life to achieve special effects.

\section{Conclusions}

It is noteworthy that, a network video works will not apply with too much variety of editing techniques and special effects, because too many and complex disorder will destroy the image showing the subject of video content, and also destroy the video sense of overall picture.

The acuisition of a certain amount of online video editing skills will make people relatively independent of the picture and sound that mixed together organically, and be more conducive to the recognition of formation system, the color of the web video and audio clips.

\section{REFERENCES}

[1] D. D. Zhao, "Breakthrough or Regression: Tail under the Online Video Advertising Market," China Advertising, September 2009.

[2] Z. H. Zhang, “The Next Step in Online Video,” Internet Weekly, 5 October 2009.

[3] H. H. Xu, “The Advantages of Online Video Advertising and Problem Analysis," Science and Technology Information, No. 9, 2010.

[4] Z. M. Zhang and Z. Liu, "Principle and Technology of Non-Linear Editing,” Tsinghua University Press, Beijing, 2008.

[5] X. Y. Lu and J. Y. Yao, "The Influence of Internet Interpersonal Communication to Relationship and Loneliness of College Students," WISM, IEEE Computer Society, 2010, pp. 387-389.

[6] X. Y. Lu, "The New Mode of the Construction of Advertising Course Based on Distance Education: The Research of the Exploitation of the Network Course of the 'Ad Design and Facture on Computer' in the National New Century Network Course Construction Project,” CSIE IEEE Computer Society, Los Angeles/Anaheim, EI Compendex, 2009, pp. 545-549.

[7] F. S. Ting, "Fang Dezhong Deal with Adobe Premiere Multimedia Elements," Chongqing Institute of Technology, Chongqing, 2008.

[8] Y.-C. Lee and Y. C. Sun, "Using Instant Messaging to Enhance the Interpersonal Relationships of Taiwanese Adolescents: Evidence from Quantile Regression Analysis,” Adolescence, Vol. 44, No. 173, 2009.

[9] NMA TV, "Matching AD Formats to How Viewers Watch Online Video," Anonymous, New Media Age, London, 2008, p. 25. 